\title{
Deep electron and hole polarons and bipolarons in amorphous oxide
}

\author{
Moloud Kaviani, ${ }^{1, *}$ Jack Strand $,{ }^{2}, \dagger$ Valery V. Afanas’ev, ${ }^{3, \ddagger}$ and Alexander L. Shluger ${ }^{1,2,}$, \\ ${ }^{1}$ Advanced Institute for Materials Research (AIMR), Tohoku University, Sendai 980-8577, Japan \\ ${ }^{2}$ Department of Physics and Astronomy and London Centre for Nanotechnology, \\ University College London, Gower Street, London WC1E 6BT, United Kingdom \\ ${ }^{3}$ Department of Physics, University of Leuven, Celestijnenlaan 200D, 3001 Leuven, Belgium
}

\begin{abstract}
Amorphous (a)- $\mathrm{HfO}_{2}$ is a prototype high dielectric constant insulator with wide technological applications. Using $a b$ initio calculations we show that excess electrons and holes can trap in a$\mathrm{HfO}_{2}$ in energetically much deeper polaron states than in the crystalline monoclinic phase. The electrons and holes localize at precursor sites, such as elongated $\mathrm{Hf}-\mathrm{O}$ bonds or under-coordinated $\mathrm{Hf}$ and $\mathrm{O}$ atoms and the polaronic relaxation is amplified by the local disorder of amorphous network. Single electron polarons produce states in the gap at $\sim 2 \mathrm{eV}$ below the bottom of the conduction band with average trapping energies of $1.0 \mathrm{eV}$. Two electrons can form even deeper bipolaron states on the same site. Holes are typically localized on under-coordinated $\mathrm{O}$ ions with average trapping energies of $1.4 \mathrm{eV}$. These results advance our general understanding of charge trapping in amorphous oxides by demonstrating that deep polaron states are inherent and do not require any bond rupture to form precursor sites.
\end{abstract}

Electron and hole states with energy levels lying deep in the bandgap impair the dielectric quality of insulating layers. In particular, electron transitions facilitated by these states account for multitude of degradation phenomena including enhanced leakage current and charge trapping eventually leading to the dielectric barrier failure and breakdown. Routinely, however, these deep electron states are seen as not inherent to the perfect material but rather associated with the presence of defects and/or impurity centers in the atomic network of an insulator. This belief offers some hope that imperfections can be eliminated by using more clean and optimized synthesis and proper processing of the insulators. On the other hand, self-trapping of excess charges in the form of small electron and hole polarons is well known to occur even in perfect crystalline oxide insulators. However, it is usually shallow, with trapping energies of the order of 0.2 $\mathrm{eV}$ (see e.g. [1-3]). As a result, the electron and hole polarons are mobile at room temperature in crystalline reduced $\mathrm{TiO}_{2}$ and $\mathrm{NiO}[4], \mathrm{CeO}_{2}[5,6]$, doped $\mathrm{ZrO}_{2}[7,8]$, and in plethora of other oxides (see e.g. [1,9-12]). The intrinsic localization of excess electrons and holes in noncrystalline materials and liquids has also been a subject of extensive experimental and theoretical studies pioneered in [13]. Structural disorder typically induces shallow electron states near the bottom of the conduction band, below the so-called mobility edge (see, e.g. [14]).

Nevertheless, evidences are starting to emerge that intrinsic electron and hole localization can occur in much deeper states in some wide gap amorphous oxides, where electrons and holes either do not self-trap or form only shallow states in the crystalline phase of the material. In amorphous $\mathrm{SiO}_{2}$, holes [15] and electrons have been

\footnotetext{
* moloud.kaviani@gmail.com

† jack.strand.14@ucl.ac.uk

$\ddagger$ valeri.afanasiev@fys.kuleuven.be

$\S$ a.shluger@ucl.ac.uk
}

shown $[16,17]$, to localize spontaneously in deep states with well-defined EPR and optical absorption signatures measured experimentally [18]. The hole trapping energies in amorphous $\mathrm{TiO}_{2}$ were calculated to be much larger than these in rutile [19]. Under-coordinated indium has been suggested to act as a deep intrinsic electron-trap center in amorphous $\mathrm{InGaZnO}_{4}$ by theoretical calculations [20]. In these systems, the polaronic relaxation is amplified by the local disorder of amorphous network. Here we turn to amorphous (a)- $\mathrm{HfO}_{2}$, which represents a wide class of high dielectric constant oxides recently emerged as the major contenders to replace $\mathrm{SiO}_{2}$ in a broad spectrum of nano-electronic devices ranging from deep-scaled transistors to DRAM and non-volatile memory cells (see, e.g. [21, 22]). Amorphous oxides make the backbone of most electronic devices and charge trapping appears to be the key factor determining device reliability. For example, accumulation of negative charge associated with deep electron trapping states observed in a- $\mathrm{HfO}_{2}$ films [23] has not yet been understood despite the clear indication that this kind of instability represents the major factor limiting lifetime of transistors [24]. We demonstrate by computational modelling that in amorphous (a)- $\mathrm{HfO}_{2}$ both electrons and holes can trap spontaneously in deep states induced by the reduced coordination and disorder of network atoms (with trapping energies exceeding $1.0 \mathrm{eV}$ ). Moreover, electrons form even deeper bipolaron states, which have not been observed before in binary oxides. The existence of these states points towards the inherent weakness of the whole amorphous oxide insulation concept.

In this class of oxides, mobile electron and hole polarons have been observed in n-doped cubic $\mathrm{ZrO}_{2}[7,8]$, which is iso-structural and has very similar properties to $\mathrm{HfO}_{2}$. Shallow electron and hole polaron states have been predicted by theoretical calculations in crystalline monoclinic (m)- $\mathrm{HfO}_{2}$ and $\mathrm{m}-\mathrm{ZrO}_{2}$ [25] and corundum [3]. Subsequent work [26] confirmed that holes can self-trap at three-coordinated $\mathrm{O}$ sites in $\mathrm{m}-\mathrm{HfO}_{2}$ and $\mathrm{ZrO}_{2}$ with 
trapping energies of about $0.2 \mathrm{eV}$ and in much deeper states at some low-coordinated $\mathrm{O}$ sites at the surface of $\mathrm{ZrO}_{2}$ [27]. Recent resonant photoelectron spectroscopy measurements strongly suggest hole polaron trapping in $\mathrm{HfO}_{2}$ thin films [28]. Thus disorder and variability of local coordination clearly play important roles in creating deep states for polaron localization.

However, $\mathrm{HfO}_{2}$, as well as other similar oxides, are not conventional glass formers and form amorphous structures in thin films due to the deposition process and substrate constraints. Such films often turn polycrystalline during anneal above $450{ }^{\circ} \mathrm{C}$ (see e.g. [29]). Models of a$\mathrm{HfO}_{2}$ structures are usually obtained using a melt-quench procedure similar to that used to create a-SiO ${ }_{2}$ structures [20, 30-33]. In this work we used this method and classical molecular dynamics accompanied by the structure relaxation using energy minimization and Density Functional Theory (DFT). LAMMPS package [34] was used with two different force-fields: relatively simple pair potentials (PPs) parametrized in [30] and a more complex charge equilibration force-field COMB [35] for comparison. In all cases, cubic periodic cells were initially equilibrated at $300 \mathrm{~K}$. The temperature was then linearly ramped to $6000 \mathrm{~K}$ at constant pressure and the structures were stabilized for $500 \mathrm{ps}$ at $6000 \mathrm{~K}$. The systems were cooled down from $6000 \mathrm{~K}$ to $0 \mathrm{~K}$ in 8 ns with a cooling rate of $0.75 \mathrm{~K} \cdot \mathrm{ps}^{-1}$. The Berendsen thermostat and barostat were used to control the simulations. In spite of relatively slow cooling rate comparing to other studies, these structures essentially correspond to frozen melt.

TABLE I. Distribution of $\mathrm{O}$ and $\mathrm{Hf}$ ion coordination numbers $(\mathrm{CNs})$ in $\mathrm{a}-\mathrm{HfO}_{2}$ periodic cells of different sizes. The numbers in brackets are from the COMB calculations. The CNs for 96 and 324 atom cells are obtained after the DFT geometry optimization of the cell volume and internal coordinates. CNs fractions are in percentage and for each ion type the sum is $100 \%$.

\begin{tabular}{ccccccc}
\hline \hline Type CN & 96 & 324 & 768 & 1500 & 6144 \\
\hline \multirow{3}{*}{ Hf } & 5 & $8.1[11.6]$ & $9.6[14.1]$ & 9.0 & 9.7 & 8.2 \\
& 6 & $47.6[48.7]$ & $65.6[60.9]$ & 78.1 & 75.5 & 75.3 \\
& 7 & $44.3[39.7]$ & $24.8[25.0]$ & 12.9 & 14.8 & 16.5 \\
\hline \multirow{3}{*}{ O } & 2 & $6.4[10.5]$ & $6.0[10.9]$ & 6.1 & 6.6 & 5.8 \\
& 3 & $69.2[65.0]$ & $83.1[71.0]$ & 85.9 & 84.3 & 84.2 \\
& 4 & $24.4[24.5]$ & $10.9[18.1]$ & 8.0 & 9.1 & 10.0 \\
\hline \hline
\end{tabular}

Both force-fields produce amorphous structures with densities of about $9.0 \mathrm{~g} \mathrm{~cm}^{-3}$ which exhibit wide distributions of bond lengths and atomic coordinations. The coordination number of each atom was determined by counting the number of atoms within a cut-off radius of $2.35 \AA$. Since periodic boundary conditions impose constraints, we checked the dependence of the structure on the cell size using PPs. The results for different cell sizes presented in Table I demonstrate the convergence for cell sizes exceeding 324 atoms. They are in qualitative agreement with other theoretical studies [33, 36, 37] and importantly do not depend significantly on the force-field used. The structures generated by the COMB force-field are similar to those produced by the activation relaxation technique [38] at the same density. However, that detailed quantitative comparison with previous studies is hampered by the fact that melt-quench methods use very different cooling rates and either predict or use different densities (ranging between 8.6 and $10.6 \mathrm{~g} \mathrm{~cm}^{-3}$ for $\mathrm{HfO}_{2}$ ). Nevertheless, all calculations show the existence of two-coordinated $\mathrm{O}$ and five-coordinated $\mathrm{Hf}$ ions, which will play an important role in our predictions. We note that the distribution of ionic coordinations is the main difference with the a- $\mathrm{SiO}_{2}$ structure where the local coordination of $\mathrm{Si}$ and $\mathrm{O}$ ions is largely preserved. Similar behaviour has been observed in other glass structures which do not contain typical network-forming cations [20, 39].

Studying the statistical distributions of properties of polarons requires extensive quantum mechanical calculations, which restrict the maximum cell size to 324 atoms. We have run exploratory calculations in 96 atom cells accompanied by further calculations in 324 atom cells. Thirty five periodic models with 96 atoms and 9 models with 324 atoms in a cell were created using PPs for this purpose. Ten 96 and 324 atom models were created using COMB and the same procedure to check the dependence of the results on the force-field used.

Further optimization of the volume and geometry of these structures was performed using DFT implemented in the CP2K code $[40,41]$ with the non-local PBE0-TCLRC functional and the exchange cutoff radius of $4.0 \AA$ [41]. The CP2K code employs a Gaussian basis set mixed with an auxiliary plane-wave basis set [42]. The double$\zeta$ Gaussian basis-sets [43] were employed on all atoms in conjunction with the GTH pseudopotential [44]. The plane-wave cutoff was set to $6530 \mathrm{eV}$ (480 Ry). To reduce the computational cost of nonlocal functional calculations, the auxiliary density matrix method (ADMM) was employed [41]. All geometry optimizations were performed using the BFGS optimizer to minimize forces on atoms to within $2.3 \times 10^{-2} \mathrm{eV} \AA^{-1}$. The trapping energies of excess electrons and holes are corrected using the method of Lany and Zunger [45, 46] using the dielectric constant of 22 [47]. The average value of a single localized charge correction for 35 structures with 96 -atom a- $\mathrm{HfO}_{2}$ supercells is $0.05 \mathrm{eV}$.

The topology of a- $\mathrm{HfO}_{2}$ models obtained using classical MD calculations and PPs does not change as a result of DFT geometry optimization of the volume and atomic structures but is different in the case of the COMB forcefield. In all cases, the optimized structures have higher densities, in the range of $9.2-9.9 \mathrm{~g} \mathrm{~cm}^{-3}$, averaging at $9.6 \mathrm{~g} \mathrm{~cm}^{-3}$. The distributions of $\mathrm{Hf}-\mathrm{O}$ and $\mathrm{Hf}-\mathrm{Hf}$ bond lengths obtained after the DFT cell and geometry optimization of neutral cells are presented in Fig. S1 in Supplementary Material [48]. The average Hf-O bond length is $2.1 \AA$ (ranging from 1.95 to $2.35 \AA$ ) is very close to the $\mathrm{Hf}-\mathrm{O}$ bond lengths in $\mathrm{m}-\mathrm{HfO}_{2}$ (around 2.1 $\AA)$. We note that the band gap of these a- $\mathrm{HfO}_{2}$ structures does not contain localized states (see Fig. S2 in Supple- 


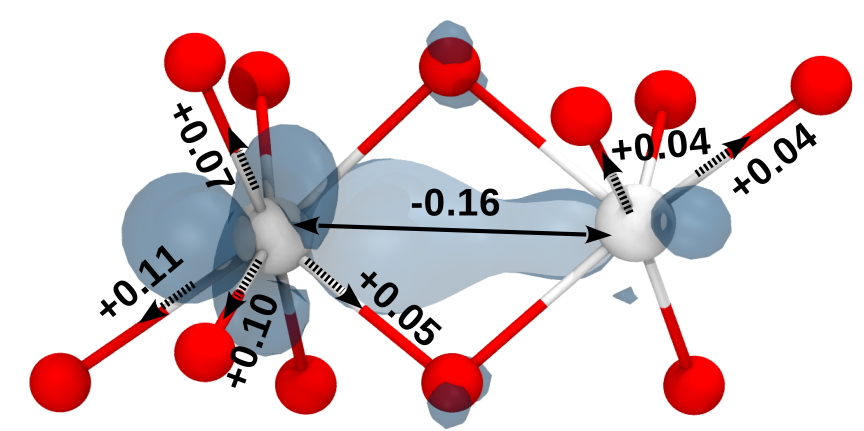

a)

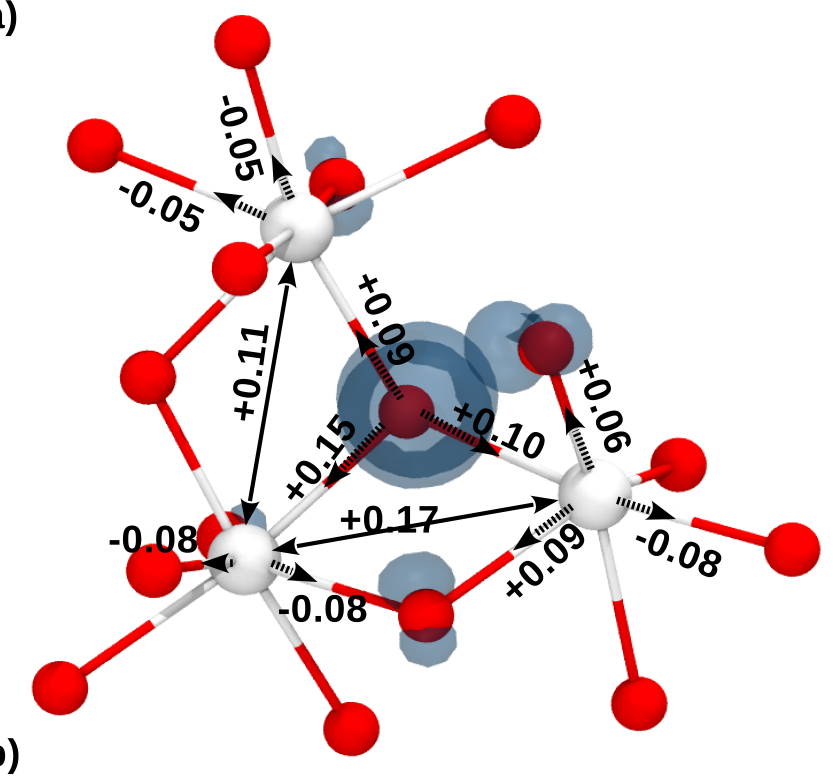

FIG. 1. Typical spin density distributions and displacements (in $\AA$ ) of $\mathrm{Hf}$ and $\mathrm{O}$ ions caused by localization of an extra electron (a) and a hole (b) in a- $\mathrm{HfO}_{2}$. The arrows show the direction of displacements. Displacements less than $0.03 \AA$ are not shown. The dashed arrows show the directions of ion displacements with respect to the neutral case. The distribution of spin density of the trapped electron (a) and hole (b) are shown in blue with different iso-value for clarity. The white spheres are $\mathrm{Hf}$ ions and the red spheres are $\mathrm{O}$ ions.

mentary material [48]). However, both the top of the valence band and the bottom of the conduction band are determined by partially localized oxygen $p$ and hafnium $d$ electronic states, similar to those observed in a- $\mathrm{SiO}_{2}$ [16]. In further calculations we compare the characteristics of excess electrons and holes localized on these states in 96 and 324 atom structures having similar densities in the range of $9.6-9.7 \mathrm{~g} \mathrm{~cm}^{-3}$.

Calculations of the electron trapping in $\mathrm{m}-\mathrm{HfO}_{2}$ show no electron localization and predict hole spontaneous selftrapping only at 3 -coordinated oxygen sites with trapping energy of $0.4 \mathrm{eV}$. The latter is calculated as the total energy difference between the delocalized hole state in the perfect lattice and the fully relaxed hole state.

Electron trapping in a- $\mathrm{HfO}_{2}$ was explored first using 35

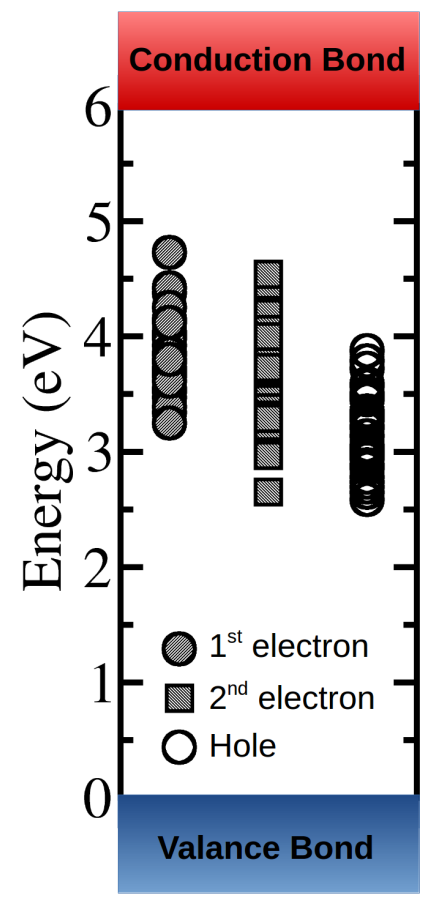

FIG. 2. Distribution of the KS levels for the electron and hole trapping in a- $\mathrm{HfO}_{2}$ structures with 324 atoms. The energy scale from 0.0 to $6.0 \mathrm{eV}$ corresponds to the a- $\mathrm{HfO}_{2}$ band gap as calculated using the PBE0-TC-LRC functional.

periodic models of a- $\mathrm{HfO}_{2}$ containing 96 atoms and then tested further using 324 atom cells. The electronic structure calculations predict an average Kohn-Sham (KS) band gap of $\sim 6.4 \mathrm{eV}$ for 96 atom cells and $\sim 6.0 \mathrm{eV}$ for 324 atom cells, ranging between 5.8 and $6.2 \mathrm{eV}$, which is in good agreement with the experimental data [28]. To study the electron trapping, an extra electron was added to these models and the geometry of each system was optimized. We observe spontaneous electron localization in deep states in each system. Unlike m- $\mathrm{HfO}_{2}$, the electron initially is not completely delocalized over the entire system, but exhibits preferential localization on some Hf atoms forming a precursor state. For example, in the structure shown in Figure 1a the excess electron is initially localized on two Hf atoms to $7 \%$ and $18 \%$ whereas the rest of the spin density is delocalized over other Hf atoms. After the geometry relaxation, the electron localization on these two Hf atoms increases to $21 \%$ and $60 \%$, respectively. Further analysis of precursor sites demonstrates that in $60 \%$ cases the extra electron is localized on the hafnium atoms which have at least three oxygen neighbors with the distance longer than $2.16 \AA$. In around $32 \%$ of the cases the extra electron is localized by the five-coordinated hafnium atoms. These Hf atoms also have longer $\mathrm{Hf}-\mathrm{O}$ bonds. In some rare cases $(\sim 8 \%)$ the extra electron is trapped on $\mathrm{Hf}$ atoms forming wide $\mathrm{O}-\mathrm{Hf}-\mathrm{O}$ angles and elongated $\mathrm{Hf}-\mathrm{O}$ bonds.

More than $80 \%$ of the electron spin density is localized 
predominantly on two or three Hf ions sharing a threecoordinated oxygen atom. Out of 35 structures, in 24 models the extra electron is localized on two neighboring Hf atoms shearing at least a three coordinated oxygen atom. In 7 models the extra electron is localized on three Hf atoms and in 4 models the extra electron is localized on four Hf atoms. The spin density is usually distributed non-homogeneously among these Hf atoms and the rest of the spin density is delocalized over other Hf atoms. The geometry optimization exposes a strong structural distortion of the $\mathrm{Hf}-\mathrm{Hf}$ and $\mathrm{Hf}-\mathrm{O}$ bonds around the electron localization site (see Fig. 1a). Typically, the Hf atoms with the localized electron displace closer (by around 0.20 A) to each other. In addition, the electron localization leads to $\mathrm{Hf}-\mathrm{O}$ bond weakening so that the $\mathrm{Hf}-\mathrm{O}$ bonds become longer on average by around $0.12 \AA$. We note that both the character of electron localization and the local network distortion around the electron trapping site are qualitatively similar to those found for an electron polaron and negatively charged oxygen vacancy in $\mathrm{m}-\mathrm{HfO}_{2}$ - the extra electron is localized by two or three Hf atoms with one of them bearing most of the spin density [25]. However, the trapping energy and the Kohn-Sham levels in the gap are much deeper in the amorphous structure.

The electron trapping energies calculated as total energy differences between the initial electron state in amorphous structure and after the geometry optimization average at $\sim 0.8 \mathrm{eV}$ with a wide distribution ranging between $0.54 \mathrm{eV}$ and $1.07 \mathrm{eV}$. These energies give a lower limit to thermal ionization energies of trapped electrons and suggest that most of these electrons will be stable at room temperature. The average position of the KS level, from the 35 models of 96 atoms, is $2.07 \mathrm{eV}$, ranging from $\sim 1.3 \mathrm{eV}$ to $2.75 \mathrm{eV}$ (see Fig. 2) below the bottom of the conduction band (CB), indicating a deep electron trap. Thus the first excess electron digs a deep potential well in the amorphous structure.

It turns out that this well can accommodate two paired electrons forming a bipolaron. The second electron is localized at the same place with a similar pattern of network distortion, making the Hf-O bonds longer by $\sim 0.09$ $\AA$ and the Hf-Hf distance shorter by $\sim 0.14 \AA$. This network relaxation facilitates the creation of a deeper singlet KS state in the gap at $\sim 2.24 \mathrm{eV}$, ranging from 1.4 to 3.4 $\mathrm{eV}$ below the bottom of the conduction band (see Fig. 2 ). For some of the structures this level is around $1.1 \mathrm{eV}$ deeper compared to the single-electron trap. This trend is similar to the bipolaron trapping in another ionic system - alkali halide melts [49-51]. It stems from the fact that the relaxation energy accompanying the second electron localization is comparable to that for the first electron due to the low density of a- $\mathrm{HfO}_{2}$, but the kinetic energy increase due to the second electron localization is much smaller as it is initially well localised in the potential well. A scaling argument for bipolaron formation in disordered media has been presented in ref. [52] and reviewed in [53]. Our results present the first structural model of such states in binary wide gap amorphous ox- ides.

We used the same approach to study hole trapping in $\mathrm{a}-\mathrm{HfO}_{2}$. The trapping of hole polarons on single $\mathrm{O}$ atoms in $\mathrm{m}-\mathrm{HfO}_{2}$ has been previously predicted theoretically [25]. Recent calculations using the cancellation of nonlinearity approach [54] predicted that holes can trap only at 3-coordinated $\mathrm{O}$ sites in the bulk of $\mathrm{m}-\mathrm{HfO}_{2}[26]$ with trapping energy of $0.18 \mathrm{eV}$ and with much larger trapping energies at surfaces, featuring two-coordinated $\mathrm{O}$ sites.

We find hole localization in all 35 models 96 atom models of a- $\mathrm{HfO}_{2}$ The energy minimisation with respect to the initial state causes a distortion in the amorphous network and leads to localisation of over $90 \%$ of the hole spin density on two $\mathrm{O}$ atoms. The distribution over the two oxygens is, however, not equal with the hole occupying predominantly one $\mathrm{O}$ atom. The characteristic atomic displacements accompanying the hole localization are shown in Fig. 1b. The average hole trapping energy is $0.7 \mathrm{eV}$, ranging between $0.4 \mathrm{eV}$ and $1.2 \mathrm{eV}$. These values are close to those found for hole trapping at different surface sites of $\mathrm{m}-\mathrm{HfO}_{2}$ [27]. The latter is not particularly surprising as the precursor sites for hole localization are low-coordinated $\mathrm{O}$ atoms in amorphous network. The distribution of the Kohn Sham levels for the hole trapping is shown in Fig. 2.

We used 324-atom cells to check the effect of the cell size on the network relaxation around the electron trapping site and to reduce the periodic image interaction. The average position of the KS level for the electron polaron in these structures is $2.10 \mathrm{eV}$ ranging from 1.63 to $2.38 \mathrm{eV}$ below the bottom of conduction band with an average trapping energy of $1.0 \mathrm{eV}$, ranging from 0.5 to $1.15 \mathrm{eV}$. The average position of the KS level for bipolaron is $1.93 \mathrm{eV}$, ranging from 1.10 to $2.60 \mathrm{eV}$ below the bottom of conduction band. The average hole trapping energy is also increased to $1.4 \mathrm{eV}$ mainly due to the larger number of atoms involved in the network distortion in a bigger cell. We also find that 324-atom cells contain up to four precursor sites for both electron and hole trapping with trapping energies distributed within about 0.8 $\mathrm{eV}$. There is a small barrier of about $0.1-0.2 \mathrm{eV}$ for electron localization at some of these sites. We assume that these sites become randomly populated by injected electrons or holes and therefore give the total distribution of trapping energies and positions of KS levels rather than these corresponding to the lowest energy states. Finally, we note that these results do not depend significantly on whether the PPs or COMB force-field were used to generate a- $\mathrm{HfO}_{2}$ structures.

To summarize, our results demonstrate that both electrons and holes can localize in a- $\mathrm{HfO}_{2}$ in deep states with the trapping energies much larger than those predicted in $\mathrm{m}-\mathrm{HfO}_{2}$. A bipolaron localization is predicted in deeper states than for single electrons. Excess electrons are localized typically on two or three Hf ions associated with longer Hf- $\mathrm{O}$ bonds or under-coordinated Hf atoms in the structures and induce strong distortion of the surround- 
ing network. The hole trapping takes place predominantly at two-coordinated $\mathrm{O}$ sites. For the experimental density of $9.6 \mathrm{~g} \mathrm{~cm}^{-3}$, the broad distribution of KS levels at 1.1-2.6 eV of polaron and bipolaron states is in a good agreement with the experimental data on exhaustive photo-depopulation spectroscopy of tapped electrons in $\mathrm{HfO}_{2}$ layers prepared using different $\mathrm{Hf}$ precursors or subjected to the post-deposition anneal $[23,55]$.

These results demonstrate that excess electrons and holes localize in crystalline and amorphous $\mathrm{HfO}_{2}$ in a qualitatively similar manner, but trapping energies in amorphous structures are much larger. They thus broaden the concept of intrinsic polaron trapping to disordered wide gap oxides. Localization of excess electrons in deep states has so far been observed in a small number of systems, such as polar $[56,57]$ and non-polar $[58,59]$ liquids, ammonia and water ice and amorphous films on metal substrates [60-62], and alkali halide melts $[49,50,63,64]$. In the latter case, bipolarons facilitated by fluctuations in the melt have also been observed and calculated [49-51], although electron polarons do not form in alkali halide crystals.

Our results may have profound implications for our understanding of charge trapping in functional ceramics. The polaron states in the gap of a- $\mathrm{HfO}_{2}$ are close to the position of the bottom of Si conduction band at $\mathrm{Si} / \mathrm{HfO}_{2}$ interface indicating that these states can be populated via direct tunnelling or electron injection into the oxide. Similar states may exist in other amorphous oxide films and nanoparticles, such as $\mathrm{Al}_{2} \mathrm{O}_{3}, \mathrm{ZrO}_{2}$, and $\mathrm{TiO}_{2}$ [19]. Finally, the revealed existence of deep intrinsic states of polaronic origin suggests a possible explanation to the abnormally high barriers often found at the interfaces between metals and high-permittivity insulating oxides such as $\mathrm{HfO}_{2}$ and $\mathrm{ZrO}_{2}$ [65]. In particular, metals with low work function $(\mathrm{Al}, \mathrm{Mg}$ ) exhibit nearly the same barrier height as in the wide-gap $\mathrm{SiO}_{2}$ despite a $1.0 \mathrm{eV}$ difference in the conduction band bottom energy position suggesting the presence of negative charges in the near-interface oxide. This effect can result from electron trapping in the deep polaron states found in the present study. More detailed understanding of these fundamental issues requires further theoretical and experimental research into polaronic states in metastable amorphous oxides.

MK and ALS are grateful to the World Premier International Research Center Initiative (WPI) sponsored by the Ministry of Education, Culture, Sports, Science and Technology (MEXT), Japan for financial support. JS and ALS acknowledge EPSRC grant EP/K01739X/1. VVA and ALS thank the EU FP7 project MORDRED (EU Project grant No. 261868) and COST Action CM1104 for financial support. Computer facilities on Archer service have been provided via the UKs HPC Materials Chemistry Consortium, which is funded by EPSRC (EP/F067496). The authors thank the Supercomputer Center, the Institute for Solid State Physics, the University of Tokyo for the facilities and the use of the SGI Altix ICE 8400EX. The authors wish to thank A.-M. El-Sayed, D. Z. Gao, T. Durrant, K. Akagi for helpful discussions and A. Foster and T. Musso for help in using COMB forcefield in LAMMPS.
[1] A. M. Stoneham, J. Gavartin, A. L. Shluger, A. V. Kimmel, G. Aeppli, C. Renner, et al., J. Phys.: Condens. Matter 19, 255208 (2007).

[2] H. Peng and S. Lany, Phys. Rev. B 85, 201202 (2012).

[3] J. B. Varley, A. Janotti, C. Franchini, and C. G. Van de Walle, Phys. Rev. B 85, 081109 (2012).

[4] I. G. Austin and N. F. Mott, Adv. Phys. 50, 757 (2001).

[5] H. L. Tuller and A. S. Nowick, J. Phys. Chem. Solids 38, 859 (1977).

[6] I. K. Naik and T. Y. Tien, J. Phys. Chem. Solids 39, 311 (1978).

[7] W. Weppner, Zeitschrift für Naturforschung A 31, 1336 (1976).

[8] J.-H. Park and R. N. Blumenthal, J. Electrochem. Soc. 136, 2867 (1989).

[9] A. Bosman and H. Van Daal, Adv. Phys. 19, 1 (1970).

[10] O. Schirmer, in Defects and Surface-Induced Effects in Advanced Perovskites (Springer, 2000) pp. 75-88.

[11] A. S. Alexandrov and J. T. Devreese, Advances in polaron physics (Springer, 2010).

[12] A. J. Rettie, W. D. Chemelewski, D. Emin, and C. B. Mullins, J. phys. chem. lett. (2016).

[13] H. Frohlich, Proc. Royal Soc. A 188, 521 (1947).

[14] N. Mott, J. Phys. C 20, 3075 (1987).

[15] D. L. Griscom, m, J. Non-Cryst. Solids 352, 2601 (2006).
[16] A.-M. El-Sayed, M. B. Watkins, V. V. Afanas'ev, and A. L. Shluger, Phys. Rev. B 89, 125201 (2014).

[17] A.-M. El-Sayed, K. Tanimura, and A. L. Shluger, J. Phys.: Condens. Matter 27, 265501 (2015).

[18] Y. Sasajima and K. Tanimura, Phys. Rev. B 68, 014204 (2003).

[19] H. H. Pham and L.-W. Wang, Phys. Chem. Chem. Phys. 17, 541 (2015).

[20] H.-H. Nahm and Y.-S. Kim, NPG Asia Mater. 6, e143 (2014).

[21] M. Houssa (Ed.), High $k$ Gate Dielectrics (CRC Press, 2003).

[22] G. He and Z. Sun (Ed.), High-k Gate Dielectrics for CMOS Technology (John Wiley \& Sons, 2012).

[23] V. V. Afanas'ev, W. C. Wang, F. Cerbu, O. Madia, M. Houssa, and A. Stesmans, ECS Trans. 64, 17 (2014).

[24] T. Ando, Materials 5, 478 (2012).

[25] D. Muñoz Ramo, A. L. Shluger, J. L. Gavartin, and G. Bersuker, Phys. Rev. Lett. 99, 155504 (2007).

[26] K. P. McKenna, M. J. Wolf, A. L. Shluger, S. Lany, and A. Zunger, Phys. Rev. Lett. 108, 116403 (2012).

[27] M. J. Wolf, K. P. McKenna, and A. L. Shluger, J. Phys. Chem. C 116, 25888 (2012).

[28] S. A. Corrêa, S. Brizzi, and D. Schmeisser, J. Vac. Sci. Technol. A 34, 01A117 (2016). 
[29] Y. B. Zheng, S. J. Wang, and C. H. A. Huan, Thin solid films 504, 197 (2006).

[30] G. Broglia, G. Ori, L. Larcher, and M. Montorsi, Model. Simul. Mater. Sci. Eng. 22, 065006 (2014).

[31] D. Vanderbilt, X. Zhao, and D. Ceresoli, Thin Solid Films 486, 125 (2005).

[32] Y. Wang, F. Zahid, J. Wang, and H. Guo, Phys. Rev. B 85, 224110 (2012).

[33] T.-J. Chen and C.-L. Kuo, J. Appl. Phys. 110, 064105 (2011).

[34] S. Plimpton, J. Comput. Phys. 117, 1 (1995).

[35] T.-R. Shan, B. D. Devine, J. M. Hawkins, A. Asthagiri, S. R. Phillpot, S. B. Sinnott, et al., Phys. Rev. B 82, 235302 (2010).

[36] S. Clima, Y. Y. Chen, R. Degraeve, M. Mees, K. Sankaran, B. Govoreanu, M. Jurczak, S. De Gendt, and G. Pourtois, Appl. Phys. Lett. 100, 133102 (2012).

[37] C. Tang and R. Ramprasad, Phys. Rev. B 81, 161201 (2010).

[38] D. Ceresoli and D. Vanderbilt, Phys. Rev. B 74, 125108 (2006).

[39] J. Akola, S. Kohara, K. Ohara, A. Fujiwara, Y. Watanabe, A. Masuno, T. Usuki, T. Kubo, A. Nakahira, K. Nitta, et al., Proc. Natl. Acad. Sci. USA 110, 10129 (2013).

[40] J. VandeVondele, M. Krack, F. Mohamed, M. Parrinello, T. Chassaing, and J. Hutter, Comput. Phys. Commun. 167, 103 (2005).

[41] M. Guidon, J. Hutter, and J. VandeVondele, J. Chem. Theory Comput. 5, 3010 (2009).

[42] G. Lippert, J. Hutter, and M. Parrinello, Mol. Phys. 92, 477 (1997).

[43] J. VandeVondele and J. Hutter, J. Chem. Phys. 127, 114105 (2007).

[44] S. Goedecker, M. Teter, and J. Hutter, Phys. Rev. B 54, 1703 (1996).

[45] S. Lany and A. Zunger, Model. Simul. Mater. Sci. Eng. 17, 084002 (2009).

[46] S. Lany and A. Zunger, Phys. Rev. B 78, 235104 (2008).

[47] C. Driemeier, R. Wallace, and I. Baumvol, ECS Transactions 11, 91 (2007).

[48] Supplementary Materials for discribing the distribution of $\mathrm{Hf}-\mathrm{O}$ and $\mathrm{Hf}-\mathrm{Hf}$ bond lengths and the Density of States of the samples after DFT cell and geometry optimization.

[49] B. Von Blanckenhagen, D. Nattland, K. Bala, and W. Freyland, J. Chem. Phys. 110, 2652 (1999).

[50] O. Terakado, P. Poh, and W. Freyland, J. Phys.: Condens. Matter 15, 1553 (2003).

[51] E. S. Fois, A. Selloni, M. Parrinello, and R. Car, J. Phys. Chem. 92, 3268 (1988).

[52] M. H. Cohen, E. N. Economou, and C. M. Soukoulis, Phys. Rev. B 29, 4500 (1984).

[53] Y. M. Galperin, V. G. Karpov, and V. I. Kozub, Adv. Phys. 38, 669 (1989).

[54] S. Lany and A. Zunger, Phys. Rev. B 81, 205209 (2010).

[55] V. V. Afanas'ev and A. Stesmans, J. Appl. Phys. 95, 2518 (2004).

[56] Y. Tang, H. Shen, K. Sekiguchi, N. Kurahashi, T. Mizuno, Y.-I. Suzuki, and T. Suzuki, Phys. Chem. Chem. Phys. 12, 3653 (2010).

[57] S. C. Doan and B. J. Schwartz, J. Phys. Chem. Lett. 4, 1471 (2013).

[58] B. S. Yakovlev, Russ. Chem. Rev. 48, 615 (1979).
[59] W. Schmidt and E. Illenberger, Nukleonika 48, 75 (2003).

[60] R. Balog, P. Cicman, D. Field, L. Feketeova, K. Hoydalsvik, N. C. Jones, T. A. Field, and J.-P. Ziesel, J. Phys. Chem. A 115, 6820 (2011).

[61] J. Stähler, U. Bovensiepen, M. Meyer, and M. Wolf, Chem. Soc. Rev. 37, 2180 (2008).

[62] J. Stähler, M. Meyer, U. Bovensiepen, and M. Wolf, Chem. Sci. 2, 907 (2011).

[63] H. Brands, N. Chandrasekhar, H. Hippler, and A.-N. Unterreiner, Phys. Chem. Chem. Phys. 7, 3963 (2005).

[64] S. Dogel, W. Freyland, H. Hippler, D. Nattland, C. Nese, and A.-N. Unterreiner, Phys. Chem. Chem. Phys. 5, 2934 (2003).

[65] V. V. Afanas'ev and A. Stesmans, J. Appl. Phys. 102, 081301 (2007) 